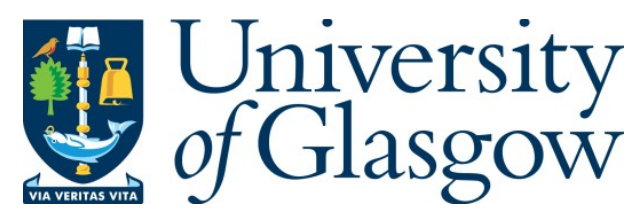

Shakthivel, D., Taube, W., Raghavan, S., and Dahiya, R. (2015) VLS Growth Mechanism of Si-Nanowires for Flexible Electronics. In: 11th Conference on Ph.D. Research in Microelectronics and Electronics (PRIME), Glasgow, Scotland, 29 Jun - 02 Jul 2015, pp. 349-352. ISBN 9781479982295.

There may be differences between this version and the published version. You are advised to consult the publisher's version if you wish to cite from it.

http://eprints.gla.ac.uk/123048/

Deposited on: 22 September 2016

Enlighten - Research publications by members of the University of Glasgow http://eprints.gla.ac.uk 


\title{
VLS Growth Mechanism of Si-Nanowires for Flexible Electronics
}

\author{
D. Shakthivel ${ }^{1}$, W. Taube ${ }^{1}$, S. Raghavan ${ }^{2}$, R. Dahiya ${ }^{1^{*}}$ \\ ${ }^{1}$ Electronics and Nanoscale Engineering, School of Engineering, University of Glasgow, G12 8QQ, UK. \\ ${ }^{2}$ Centre for Nano Science and Engineering, Indian Institute of Science, Bangalore-560012, India. \\ *Correspondence to: Ravinder.Dahiya@glasgow.ac.uk
}

\begin{abstract}
Nanowires (NWs) are promising building blocks for flexible electronics and sensors and a number of approaches have been used to develop them. Among these, the vapor-liquidsolid (VLS) mechanism has been most appealing as it provides the electronic quality NWs at low fabrication cost. For these reasons, this method plays an important role in many applications including NWs based flexible electronics. The performance of NWs based electronics and sensors depend on their quality and the underlying growth mechanism, which thus far has not attracted much attention. In this paper, we present the physical chemistry model that explains the atomistic aspects of the growth mechanism of silicon nanowires. The mechanistic equations have been derived for various steps involved in a standard VLS growth process. The supersaturation under the steady state conditions has been calculated and utilized to estimate the growth rate of Si-NWs under different temperature conditions. The estimated values are found to be consistent with the reported measured values. The results from our study indicate that the $\mathrm{Si}-\mathrm{NW}$ growth rate is directly related to the temperature. High-temperatures $\left(\sim 900^{\circ} \mathrm{C}\right)$ lead to longer Si-NWs (tens of microns length). This knowledge about growth conditions for Si-NW will enable better control of Si-NW dimensions and hence will have significant positive impact on using Si-NW in flexible electronics - especially the contact printing of NWs based electronic layers on flexible substrates.
\end{abstract}

Keywords-Flexible elecronics, Silicon nanowires, VLS mechanism, Kinetic model.

\section{INTRODUCTION}

Electronics on flexible substrates is the burgeoning field which will enable numerous exciting applications such as electronic or tactile skin, wearable electronics, smart patches, flexible displays etc. [1-5]. A number of approaches, using a range of organic/inorganic materials and various fabrication techniques including printing of electronics are being explored now-a-days for realizing flexible electronics. A large number of active and passive devices such as light emitting diodes (LEDs), solar cells, sensors, etc. have been fabricated on flexible substrates $[6,7]$. Among the various approaches, $\mathrm{Si}$ micro/nanostructures for flexible electronics are very promising. . The high-mobility and inherent flexibility, owing to small size, makes micro/nanostructures such as single crystal Si-NWs (1D nanostructures with diameters ranging from 3 to $100 \mathrm{~nm}$ [8]) very attractive for high-performance electronics on large areas and flexible substrates. At nanoscale, Si-NWs exhibits interesting electrical, optical, mechanical and electrochemical properties [9], which have been exploited to develop nano-electronics [10], sensors [11], and photovoltaic energy harvesting [9] devices. An added advantage of $\mathrm{Si}-\mathrm{NW}$ approach over other material systems is the compatibility with CMOS technology.

Si-NWs used in the above applications are either realized using top-down approach based on conventional lithography and etching technique or they are synthesized atom-by-atom using bottom-up strategy [8,9]. The top-down approaches typically employ sophisticated tools to fine tune the NW dimensions. The crystal structure and composition of the NWs in top-down approach is same as the mother wafer. Contrary to this, the bottom-up methods allow materials engineering to develop complex hetero-structures such as $\mathrm{Ge} / \mathrm{Si}$ core-shell NWs that (owing to changed band-gap) are transparent to holes, and hence lead to ohmic junctions and improved performance of electronic devices. Therefore, the possibility to engineer the structures is a significant gain from the viewpoint of high-performance flexible electronics. Further, the cost of fabrication of NWs is lower in the case of bottom-up approach. The Si-NWs in bottom-up approach are synthesized using catalyst particle assisted vapour-liquid-solid (VLS) mechanism. The VLS mechanism yields most pristine and single crystalline NWs from $3 \mathrm{~nm}$ to tens of micron diameter $[8,12]$.

From applications viewpoint, the Si-NWs are harvested either from mother wafers or from growth substrates and transferred to the final flexible substrate using printing or stamping techniques. A large number of devices such as photo detector, sensors, NMOSFET, ring oscillators and differential amplifiers have been reported with transfer printing of $\mathrm{Si}$ micro/nanostructures [13-17]. Large area array of NWs over flexible substrates have been demonstrated for pressure sensor application which potentially leads to the fabrication of highly sensitive artificial skin [18]. Despite the complexities involved in the transfer and assembling mechanisms [19], the NW growth process itself plays a key role in the technology development.

To gain control over the performance of electronic devices from Si-NWs it is essential to control the NW dimensions, crystallinity, dopant concentration and site specific growth. For control of all of these parameters it is important to understand the underlying mechanism for growth of Si-NWs and that of the VLS growth mechanism. In this paper, we present the physical chemistry model that explains the atomistic aspects of the growth mechanism of Si-NWs. 
This paper is organized as follows: The VLS growth mechanism using chemical vapour deposition (CVD) is presented in section II. The results from the physical chemistry model are discussed in section III. Finally, the conclusions and the future scope are given in section IV.

\section{VLS GROWTH MECHANISM USING CVD}

\section{A. Au-Si phase diagram and thermodynamic driving force}

Among various techniques for NW synthesis, CVD assisted VLS growth of Si-NWs is widely adopted due to its versatility and wide applicability to many materials systems [8]. The ability to produce electronic quality NWs further enhances the utility of CVD technique in comparison with solution based processes. In VLS growth, the nano-sized catalyst particles are kept at an elevated temperature above eutectic point. The vapour source of the semiconducting material is allowed to be adsorbed by the catalyst particles which eventually result in saturation. At supersaturation, there is enough chemical potential present in the vapour phase to let solid wire precipitate out from the catalyst droplet/substrate interface. The size of the NWs is usually governed by the diameter of the catalyst particles. The VLS process has been utilized to synthesise NWs with diameter varying from $3 \mathrm{~nm}$ to several microns [12]. The $\mathrm{Au}$ catalyst assisted NW synthesis process could be conveniently explained using an Au-Si alloy phase diagram. Phase diagrams are graphical representations of the physical state of the $\mathrm{Au}-\mathrm{Si}$ particle at different temperatures. Different regions are separated by lines or curves. Typically, the phase diagrams have temperature on the $\mathrm{y}$-axis and $\mathrm{Au} / \mathrm{Si}$ composition ratio on the $\mathrm{x}$-axis.

The choice of the catalyst material and experimental conditions can be determined using phase diagrams [8]. Phase diagrams provide the temperature zone in which the VLS experiment can be performed for NW growth. Typically, AuSi particle of composition 19 atom $\% \mathrm{Si}$ and 81 atom \% $\mathrm{Au}$ melts at a temperature of $363^{\circ} \mathrm{C}$. Conceptually, VLS growth occurs at any temperature above $363^{\circ} \mathrm{C}$. At a given temperature, adding excess $\mathrm{Si}$ to the $\mathrm{Au}-\mathrm{Si}$ liquid solution beyond the equilibrium concentration tends to nucleate $\mathrm{Si}$ solid material. The $\mathrm{Si}$ concentration in the Au-Si solution in excess of the equilibrium quantity is the effective supersaturation driving the NW growth. It can be expressed mathematically as [20]

$$
\Delta \mu_{L S}=k T \ln \left(\frac{C}{C^{o}}\right)
$$

Where, $C$ is the concentration (in atoms $/ \mathrm{m}^{3}$ ) of $\mathrm{Si}$ in the $\mathrm{Au}$ droplet, $C^{o}$ is the equilibrium concentration which can be obtained from the phase diagram. Under the experimental conditions, the growth rate of NWs depends on the supersaturation. Estimating this fundamental parameter requires measuring of $\mathrm{Si}$ concentration in nano-sized droplet. To date no experimental effort has been successful in achieving this task. Also, for successful prediction of growth rate and experimental dependencies, it is essential to understand the kinetic aspects of VLS mechanism.

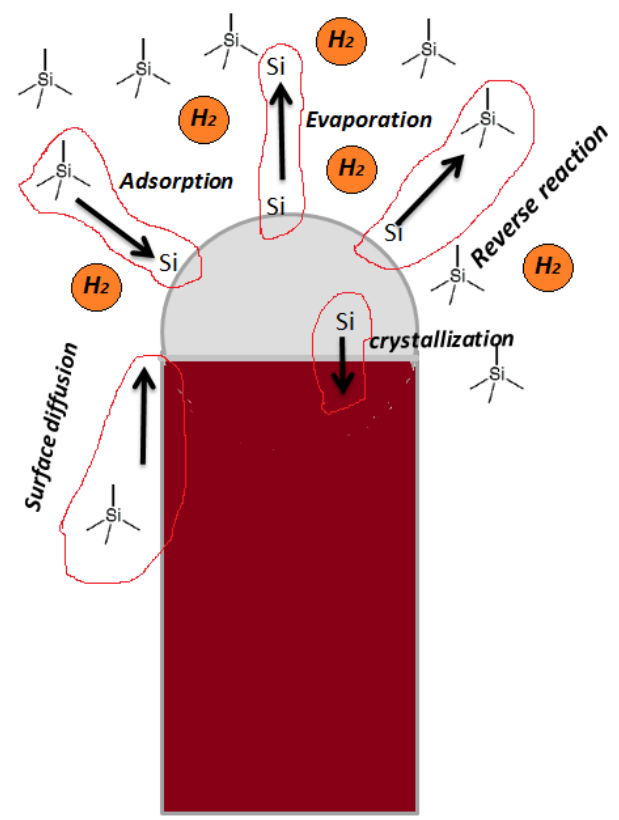

Fig. 1. Schematic diagram of VLS process for Si-NW growth.

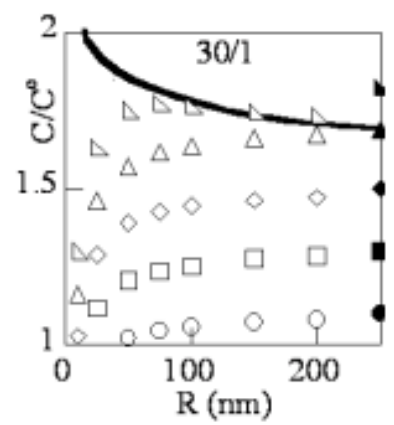

Fig. 2. The variation of $\mathrm{Si}$ supersaturation with respect to catalyst size. Each curve represents a particular vapour phase supersaturation along with a set of kinetic parameters.

\section{B. Kinetics of VLS mechanism: Au catalyzed Si-NW growth}

The growth rate of Si-NWs varies with diameter of $\mathrm{Au}$ catalyst particles [21]. This was observed and reported diameters of catalysts in the range from 10 to $100 \mathrm{~nm}$. However, in contrary to the above, recent in-situ experimental observations have shown that the growth rates remains constants in this range [22]. The kinetic models reported in the past could not explain this discrepancy. Also, the debate was centred on to identifying the step, which determines the rate of VLS mechanism [8]. Based on the experimental observations, many authors concluded that either the incorporation or the crystallization steps could be the rate controlling step [21, 23]. Recent qualitative model [24] suggested that the interplay between the crystallization and the incorporation steps at the steady state determines the rate of the VLS growth process. This model further indicated that at steady state, net rate of injection will be equal to the rate of ejection as a natural outcome. 
In this view, the developed kinetic model [25] sheds light on the atomistic nature of the VLS process. The schematic illustration of the VLS process with all the atomistic steps is given in Fig.1. In the vapour phase the precursor molecule such as $\mathrm{SiCl}_{4}$ or $\mathrm{SiH}_{4}$ are transported to the catalyst at the wire tip. Chemical reaction takes place at the surface of the droplet which results in the injection of $\mathrm{Si}$ atoms into the catalyst particle. Alternatively, $\mathrm{Si}$ atoms adsorbed at some other sites also get injected into the catalyst droplet by surface diffusion. These injected $\mathrm{Si}$ atoms further diffuse inside the catalyst droplet and reach the catalystsubstrate interface. Depending on the process temperature, some $\mathrm{Si}$ atoms leaves the droplet due to evaporation. Under certain ambient conditions, the $\mathrm{Si}$ atoms also gets ejected out of the catalyst droplet due to the reverse reaction with the gases. Out of the various processes the most important process for nanowire growth is the ejection of silicon atoms by crystallization of the atoms at the catalyst-substrate interface.

The above process is explained mathematically through Eq 2 , which indicates that the rates of injection and ejection is equal at steady-state [25].

$$
\left[\frac{d C}{d t}\right]_{\text {Injection }}=\left[\frac{d C}{d t}\right]_{\text {ejection }}
$$

Where, $C$ is the concentration of atoms (in number per unit volume) in the catalyst droplet and $t$ represents time. By including all the injection and ejection steps, Eq 2 can be written as:

$$
\begin{aligned}
& {\left[\frac{d C}{d t}\right]_{\text {Adsorption }}+\left[\frac{d C}{d t}\right]_{\text {surfacediffusion }}=} \\
& {\left[\frac{d C}{d t}\right]_{\text {evaporation }}+\left[\frac{d C}{d t}\right]_{\text {reverse }}+\left[\frac{d C}{d t}\right]_{N \text { Wgrowth }}}
\end{aligned}
$$

The rate of change in the concentration of the droplet caused by the surface adsorption of injected $\mathrm{Si}$ atoms can be treated using Langmuir-Hinshelwood kinetics, which is given by

$$
\left[\frac{d C}{d t}\right]_{\text {Adsorption }}=\left[\frac{K_{1} K_{2} P}{R}\right]_{\text {droplet }}
$$

Where $K_{l}$ is a temperature dependent constant and includes the surface reaction rate constant and the ratio of adsorption and desorption rate constants, $K_{\text {adsorption }} / K_{\text {desorption. }}$. It has units of $m / J-s$. The value of $K_{l}$ incorporates the energy barrier which the Si bearing species overcome to inject $\mathrm{Si}$ into the droplet. $K_{2}$ is a geometrical constant. Similarly, the expressions for the remaining terms have been derived and are given in [25].
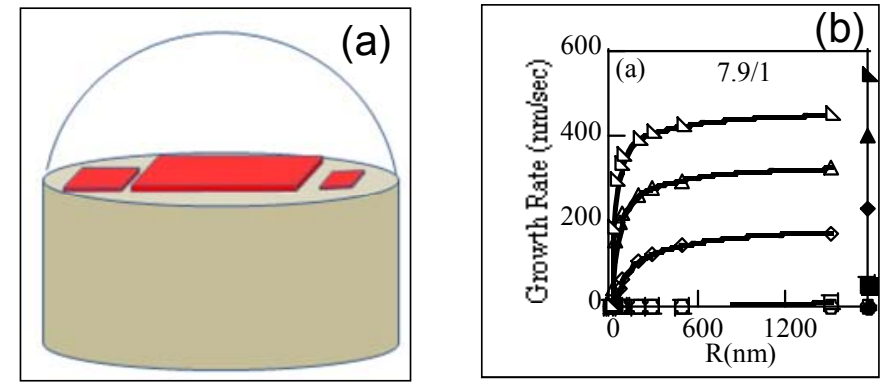

Fig.3. (a) Schematic diagram of the birth and spread layer by layer(LL) mechanism. (b) Catalyst diameter versus growth rate for layer by layer growth mode. The values of the growth rate fall within an order of the experimentally measured

\section{RESUlts AND Discussion}

\section{A. Estimation of the Si concentration in various droplets:}

The estimation of $\mathrm{Si}$ concentration is one of the core requirements of the kinetic model. We have achieved this by equating all the injection and ejection steps appropriately. As expected, the Si concentration is found to vary with the droplet size (Fig.2). The Si concentration has been estimated for two different growth modes and described in the next section. For multilayer growth mode, Si concentration remains constant irrespective of the droplet size. This justifies the growth velocity saturation observation by many authors. Also, the plots in Fig.2 determines the region at which the growth mode changes from layer by layer to multilayer. The evaluation of the growth rates by making use of the supersaturation value is explained in the next section.

\section{B. Evaluation of $N W$ growth rates:}

The NW growth rates have been estimated for different growth modes and various $\mathrm{Au}-\mathrm{Si}$ catalyst particle sizes. The growth mode of the Si-NWs has been predicted and observed in-situ as layer by layer mode (Fig. 3a). It could either be layer by layer or multilayer mode depending on the conditions and droplet diameter. by,

The growth rate of the NW in layer by layer mode is given

$$
\left(\frac{d h}{d t}\right)_{L L-I F}=\left(J \pi R^{2}\right) a
$$

Where, $R$ is wire radius, $a$ is the layer step height, and $J$ is number of nuclei formed per unit area. $J$ incorporates the supersaturation term and the desolvation energy barrier. The desolvation energy barrier reflects the energy needed to detach $\mathrm{Si}$ from Au-Si solution and to attach it over the NW lattice.

The estimated values of $\mathrm{Si}$ supersaturation have been utilized to predict the growth rate of the NWs. The growth rate values (Fig. 3b) have been calculated using a range of adsorption and desolvation energy barriers due to the nonavailability of specific data in the literature. The estimated values of the growth rates fall within an order of the measure 
ones. The growth rate versus droplet diameter data validates the kinetic model in terms of the approach.

The physical chemistry model presented above incorporates all the atomistic steps of the VLS mechanism to elucidate the growth rate dependencies. The calculated growth rates are very much within the order of the reported values. Prediction of the growth rate is important when designing NW synthesis experiments for transfer printing process. For example, for the contact printing process it is required to grow straight NWs having lengths of the order of microns and this can be achieved with controlled growth rate using VLS process. Similarly, hetero-structures such as Si-Ge core-shell structures have shown interesting properties such as transparency to charge carrier which can be useful in realizing high-performance flexible electronics. The synthesis conditions for such structures could be predicted by using the kinetic model presented here. Importantly, the adsorption and surface diffusion of Ge atoms over the Si NW surface leads to formation of the shell which could be quantified using the model. Similarly, in-situ doping concentration could be estimated using the kinetic model equations. This will enable tuning of the electrical properties of NWs.

\section{CONCLUSIONS}

The comprehensive model for the VLS process explained the discrepancies of the existing literature. The thermodynamic driving force concept is revisited and evaluated in this model. The atomistic aspects of the VLS mechanism is explained in detail which could be further extended for compound semiconductor NW growth. Among all, the study could find useful application for the synthesis of abrupt semiconductor nanowire heterojunctions. Such heterostructured NW finds significant application in high performance flexible electronics. Estimating the $\mathrm{Si}$ concentration is also important as it provides valuable insight into the state of the catalyst when the NW is growing at a constant velocity. Our model successfully estimates $\mathrm{Si}$ concentration in the catalyst droplet which is otherwise difficult to measure. This will help us tailor the catalyst composition for the abrupt heterojunctions. For example, a stack of NWs could find significant application in 3D flexible electronics. A detailed understanding of NW growth is essential if the spontaneous process that gives rise to nanowires is to be used for reliable manufacture of electronic devices, sensors etc.

\section{ACKNOWLEDGEMENT}

This work was supported by Engineering and Physical Sciences Council (EPSRC) Fellowship for Growth - Printable Tactile Skin (EP/M002527/1).

\section{REFERENCES}

[1] Y. Sun and J. A. Rogers, "Inorganic semiconductors for flexible electronics," Adv. Mater, vol. 19, pp. 1897-1916, 2007.

[2] R. S. Dahiya, "Epidermal Electronics: Flexible Electronics for Biomedical Application," in Handbook of Bioelectronics - Directly interfacing electronics and biological systems, S. Carrara and K. Iniewski, Eds., ed: Cambridge University Press, 2015, pp. 245-255.

[3] S. Khan, S. Tinku, R. S. Dahiya, and L. Lorenzelli, "Flexible Tactile Sensors using Screen Printed P(VDF-TrFE) and MWCNT/PDMS Composites," IEEE Sensors J., vol. PP, pp. 1-1, 2014.

[4] R. S. Dahiya, P. Mittendorfer, M. Valle, G. Cheng, and V. Lumelsky, "Directions Towards Effective Utilization of Tactile Skin -- A Review," IEEE Sensors J., vol. 13, pp. 4121 - 4138, 2013.

[5] R. S. Dahiya and M. Valle, Robotic Tactile Sensing - Technologies and System. Dordrecht: Springer, 2013.

[6] P. F. Moonen, I. Yakimets, and J. Huskens, "Fabrication of Transistors on Flexible Substrates: from Mass-Printing to High-Resolution Alternative Lithography Strategies," Adv. Mater, vol. 24, pp. 5526-5541, 2012.

[7] S. Khan, R. Dahiya, and L. Lorenzelli, "Technologies for Printing Sensors and Electronics over Large Flexible Substrates: A Review," IEEE Sensors J., vol. PP, pp. 1-1, 2014.

[8] V. Schmidt, J. V. Wittemann, and U. Gösele, "Growth, Thermodynamics, and Electrical Properties of Silicon Nanowires," Chem. Rev, vol. 110, pp. 361-388, 2010.

[9] N. P. Dasgupta, J. Sun, C. Liu, S. Brittman, S. C. Andrews, J. Lim, et al., "25th anniversary article: semiconductor nanowires-synthesis, characterization, and applications," Adv. Mater., vol. 26, pp. 2137-2184, 2014.

[10] W. Lu and C. M. Lieber, "Nanoelectronics from the bottom up," Nat. Mater, vol. 6, pp. 841-850, 2007.

[11] Y. Cui, Q. Wei, H. Park, and C. M. Lieber, "Nanowire Nanosensors for Highly Sensitive and Selective Detection of Biological and Chemical Species," Science, vol. 293, pp. 1289-1292, 2001.

[12] R. S. Wagner and W. C. Ellis, "Vapor-Liquid -Solid Mechanism of Single Crystal Growth," Appl. Phys. Lett, vol. 4, pp. 89-90, 1964.

[13] A. Nathan, B. k. Park, Q. Ma, A. Sazonov, and J. A. Rowlands, "Amorphous silicon technology for large area digital X-ray and optical imaging," Microelectron. Reliability, vol. 42, pp. 735-746, 2002.

[14] R. S. Dahiya, A. Adami, C. Collini, and L. Lorenzelli, "Fabrication of single crystal silicon mirco-/nanostructures and transferring them to flexible substrates," Microelectron. Eng, vol. 98, pp. 502-507, 2012.

[15] K. Zhang, J. H. Seo, W. Zhou, and Z. Ma, "Fast flexible electronics using transferrable silicon nanomembranes," J. Phys. D: Appl. Phys, vol. 45, p. 143001, 2012.

[16] M. A. Meitl, Z. T. Zhu, V. Kumar, K. J. Lee, X. Feng, Y. Y. Huang, et al., "Transfer printing by kinetic control of adhesion to an elastomeric stamp," Nat. Mater, vol. 5, pp. 33-38, 2006.

[17] T. I. Kim, Y. H. Jung, H. J. Chung, K. J. Yu, N. Ahmed, C. J. Corcoran, et al., "Deterministic assembly of releasable single crystal silicon-metal oxide field-effect devices formed from bulk wafers," Appl. Phys. Lett, vol. 102, p. 182104, 2013.

[18] K. Takei, T. Takahashi, J. C. Ho, H. Ko, A. G. Gillies, P. W. Leu, et al., "Nanowire active-matrix circuitry for low-voltage macroscale artificial skin," Nat. Mater, vol. 9, pp. 821-826, 2010.

[19] M. Kwiat, S. Cohen, A. Pevzner, and F. Patolsky, "Large-scale ordered 1D-nanomaterials arrays: Assembly or not?," Nano Today, vol. 8, pp. 677-694, 2013.

[20] I. Markov, "Crystal Growth for Beginners: Fundamentals of Nucleation," Crystal Growth and Epitaxy, World Scientific Singapore, 1995.

[21] E. Givargizov, "Fundamental aspects of VLS growth," J. Cryst. Growth, vol. 31, pp. 20-30, 1975.

[22] S. Kodambaka, J. Tersoff, M. Reuter, and F. Ross, "Diameterindependent kinetics in the vapor-liquid-solid growth of Si nanowires," Phys. Rev. Lett, vol. 96, p. 096105, 2006.

[23] G. Bootsma and H. Gassen, "A quantitative study on the growth of silicon whiskers from silane and germanium whiskers from germane," $J$. Cryst. Growth, vol. 10, pp. 223-234, 1971.

[24] V. Schmidt, S. Senz, and U. Gösele, "Diameter dependence of the growth velocity of silicon nanowires synthesized via the vapor-liquidsolid mechanism," Phys. Rev. B, vol. 75, p. 045335, 2007.

[25] D. Shakthivel and S. Raghavan, "Vapor-liquid-solid growth of $\mathrm{Si}$ nanowires: A kinetic analysis," J. Appl. Phy., vol. 112, p. 024317, 2012. 